\title{
ASYMPTOTIC VARIATIONAL FORMULAE \\ FOR EIGENVALUES
}

\author{
C. A. Swanson \\ (received March 25, 1962)
}

1. Introduction. The eigenvalues of a second order self-adjoint elliptic diffe rential ope rator on Riemannian $n$-space $R$ will be considered. Our purpose is to obtain asymptotic variational formulae for the eigenvalues under the topological deformations of (i) removing an $\varepsilon$-cell (and adjoining an additional boundary condition on the boundary component the reby introduced); and (ii) attaching an $\varepsilon$-handle, valid on a half-open interval $0<\varepsilon \leq \varepsilon$. In particular the formulae will exhibit the non-analytic nature of the variation. Similar variational problems for singular ordinary differential operators have been considered by the writer in [3] and [4].

The variation of harmonic Green's functions and other domain functionals on finite Riemann 2-surfaces has been considered at length by M. Schiffer and D. C. Spencer in their book [7]. This elegant theory depends on analytic function theory and most of the results a re written in complex form. Our treatment depends on the theory of elliptic differential equations [2] and functional analysis, and has the advantage that the results a re obtained for $n \geq 2$ and for differential equations more general than Laplace's equation. Even in the case of the Laplacian operator on finite 2-surfaces, our results are not readily available in the lite rature.

The first theorem gives a general a symptotic variational formula, which in particular can be applied to deformations of the type (i) and (ii) above. This formula is in effect a reformulation of Green's symmetric identity. To apply it

This research was supported by the United States Air Force Office of Scientific Research, under contract number AF-AFOSR-61-89.

Canad. Math. Bull. vol. 6, no. 1, Janua ry 1963. 
to the cases (i) and (ii) we shall use some uniform asymptotic estimates for eigenfunctions which were obtained in [1]. The main results a re given in theorems 3 and 5 .

2. Preliminaries. Let $M$ be an open, connected domain with compact closure in $\mathrm{R}$ whose boundary $\mathrm{B}$ consists of smooth (n-1)-dimensional closed manifolds. The latter are supposed to be homeomorphic images of the unit $(n-1)$-sphere in Euclidean space, with continuous unit normal vectors. We do not exclude the possibility that $M$ is a closed Riemannian space, that is, $B$ is void. Let $\Delta$ denote the Laplacian operator on $M$ and let $a: p \rightarrow a(p)$ denote a continuous, positive-valued function on $M$. Eigenvalue problems will be considered for the formally self-adjoint elliptic differential operator $L$ defined by

$$
(L f)(p)=-(\Delta f)(p)+a(p) f(p), \quad p \in M, \quad f \in C^{2}[M] .
$$

The basic domain $D$ is defined to be the set of all complex-valued functions on $\bar{M}$ which are of class $C^{2}[M]$, continuous on $\bar{M}$, and zero on $B$, (the last condition being deleted in the case that $B$ is void). The basic eigenvalue problem for $L$ is

$$
L x=\lambda x, \quad x \in D \text {. }
$$

Our purpose is to derive asymptotic variational formulae for the eigenvalues $\lambda$ of $L$ when the domain $D$ is perturbed to a "slightly different" domain $\mathrm{D}_{\varepsilon}\left(\right.$ or $\left.\mathrm{D}_{\varepsilon}^{*}\right)$ by the deformation of removing an $\varepsilon$-cell (or attaching an $\varepsilon$-handle) to $M$.

Let $s(p, q)$ denote the geodesic distance in $M$ from $p$ to $q$, uniquely determined for $q$ in some neighbourhood of $p[2]$. Let $q_{j}(j=1,2, \ldots J)$ be fixed but a rbitrary points in $M$. The specific $\varepsilon$-cells to be considered are the open balls $\mathrm{N}_{\varepsilon j}$ defined by

$$
N_{\varepsilon j}=\left\{p \in R: s\left(p, q_{j}\right)<\varepsilon\right\}, 0<\varepsilon \leq \varepsilon_{0} ; j=1,2, \ldots J .
$$

It will be supposed that the positive number $\varepsilon_{0}$ has been 
selected so that (i) $N_{\varepsilon j} \subset M$, and (ii) the boundary $\gamma_{\varepsilon j}$ of $\mathrm{N}_{\varepsilon j}$ is a smooth homeomorphic image of the unit $(n-1)-s p h e r e$ in Euclidean space, whenever $0<\varepsilon \leq \varepsilon_{0}(j=1,2, \ldots J)$. The parameter $\varepsilon$ measures the smallness of $\mathrm{N}_{\varepsilon j}$, and as $\varepsilon \rightarrow 0, N_{\varepsilon j}$ shrinks to the point $q_{j}$.

The notations

$$
\gamma_{\varepsilon}=\underset{j=1}{J} \gamma_{\varepsilon j}, \quad N_{\varepsilon}=\underset{j=1}{\cup} N_{\varepsilon j}, \quad M_{\varepsilon}=M-\bar{N}_{\varepsilon}
$$

will be used. The domain $\mathrm{D}_{\varepsilon}^{0}$ is defined to be the set of all complex-valued functions on $\bar{M}_{\varepsilon}$ which are of class $C^{2}\left[\mathrm{M}_{\varepsilon}\right]$, continuous on $\bar{M}_{\varepsilon}$, and zero on $B \cup \mathbb{N}_{\varepsilon}$. The notations $($, and $\|||$ will designate the inner product and norm in the Hilbert space $L^{2}[M]$.

The following lemma is an easy consequence of Green's symmetric identity for $L$ on $M_{\varepsilon}$ [2]. The unit positive normal $\underline{n}$ to $\gamma_{\varepsilon}$ is supposed to point toward the outside of $\mathrm{Y}_{\varepsilon}$ (inside of $\mathrm{M}_{\varepsilon}$ ).

LEMMA. If $u \in D_{\varepsilon}^{0}, v \in D_{\varepsilon}^{0}$, then

$$
\begin{aligned}
& (u, L v)-(L u, v)=I_{\varepsilon}[u, v], \underline{\text { where }}{ }^{1} \\
& I_{\varepsilon}[u, v]=\int_{\gamma_{\varepsilon}}(u \nabla v-\bar{v} \nabla u) \cdot \underline{n} d S
\end{aligned}
$$

3. The.main variational formula. The asymptotic variational formula (3.1) below is to be applied in the sequel

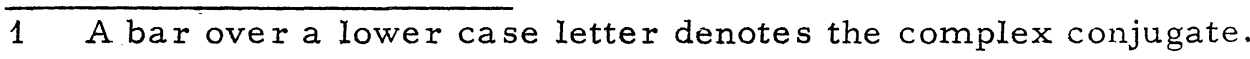


to the non-analytic surface deformations referred to in the introduction. The form of (3.1) is somewhat similar to Hadamard's classical formula [7, p. 274]. The latter is essentially an analytic formula, however, and is not pertinent to situations in which the basic and perturbed regions a re of different topological types.

THEOREM 1. Let $\lambda$ be an eigenvalue of the basic problem and let $x$ be an a rbitrary eigenfunction associated with $\lambda$. Let $\mu$ be a complex number such that the re exists a non-zero $y \in D_{\varepsilon}^{0}$ satisfying $L y=\mu y$ and $\|y-x\| \leq \delta\|x\|$, where $0<\delta \leq \delta_{0}<1$. Then

$$
\bar{\mu}-\lambda=\|x\|^{-2} I_{\varepsilon}[x, y][1+0(\delta)] \text {. }
$$

Proof. Let $\mathrm{u}$ be the function with support $\bar{M}_{\varepsilon}$ that coincides with $x$ on $\bar{M}_{E}$. Since $x \in D$, it follows that $u \in D_{\varepsilon}^{O}$. We can then apply the lemma to $u$ and $y$ to obtain

$$
\bar{\mu}(u, y)-\lambda(u, y)=(u, L y)-(L u, y)=I_{\varepsilon}[u, y] .
$$

However, $(u, y)=(x, y)$ and $u(p)=x(p)$ for $p \in \gamma_{\varepsilon}$. Then

$$
(\bar{\mu}-\lambda)(x, y)=I_{\varepsilon}[x, y] .
$$

By hypothesis, $|(y, x)-(x, x)|=|(y-x, x)| \leq \delta\|x\|^{2}$, and $|(y, x)| \geq\|x\|^{2}-|(y-x, x)| \geq\|x\|^{2}-\delta\|x\|^{2}=(1-\delta)\|x\|^{2}$.

Hence (3.2) yields

$$
\begin{aligned}
\left|(\bar{\mu}-\lambda)-\|x\|^{-2} I_{\varepsilon}[x, y]\right| & =\left|\frac{(y, x)-(x, x)}{(y, x)(x, x)} I_{\varepsilon}[x, y]\right| \\
& \leq \frac{\delta}{1-\delta}\|x\|^{-2}\left|I_{\varepsilon}[x, y]\right| .
\end{aligned}
$$

4. Asymptotic variation under cell removal. In this section $N_{\varepsilon}$ will be specialized to a single open ball $N_{\varepsilon 1}$ with centre $q_{1} \in M$ and boundary $\gamma_{\varepsilon}$. We define the perturbed 
domain $D_{\varepsilon}$ to be the set of all $f \in D_{\varepsilon}^{O}$ which vanish on $\gamma_{\varepsilon}$, and consider the perturbed eigenvalue problem

$$
\text { Ly }=\mu y, \quad y \in D_{\varepsilon} \text {. }
$$

The eigenvalues will be denoted by $\mu_{i}\left(0<\mu_{1} \leq \mu_{2} \leq \ldots\right)$ and a corresponding orthonormal set of eigenfunctions by $y_{i}$ $(i=1,2, \ldots)$.

An L-measure for $M_{\varepsilon}$ with respect to the boundary components $B$ and $Y_{\varepsilon}$ is defined to be the uniquely-determined solution $h$ of the Dirichlet problem [2]

$$
(L h)(p)=0, \quad p \in M_{\varepsilon} ; h(p)=0, p \in B ; h(p)=1, p \in \gamma_{\varepsilon} .
$$

Let $\varphi$ be the positive-valued function on $0<\varepsilon \leq \varepsilon$, defined as follows:

$$
\begin{aligned}
\varphi(\varepsilon) & =-1 / \log \varepsilon & & \text { if } \mathrm{n}=2 \\
& =\varepsilon^{\mathrm{n}-2} & & \text { if } \mathrm{n} \geq 3
\end{aligned}
$$

Except for a multiplicative constant, $\varphi$ is the reciprocal of the parametrix [2]. Estimates of the type stated in the following theorem were obtained in [1].

THEOREM 2. Corresponding to each eigenvalue $\lambda$ of the basic problem (2.1), of multiplicity $\mathrm{m}$, there are positive constants $\varepsilon_{1}$ and $c$ (independent of $\varepsilon$ ) such that exactly $m$ eigenvalues $\mu_{i}$ of (4.1) lie in the interval $[\lambda, \lambda+c \varphi(\varepsilon)]$ $\underline{\text { provided }} 0<\varepsilon \leq \varepsilon_{1}$. If $y_{1}, y_{2}, \cdots$ are orthonormal eigenfunctions associated with $\mu_{1}, \mu_{2}, \ldots$, there exists an orthonormal set $x_{1}, x_{2}, \ldots, x_{m}$ in the eigenspace of $\lambda$ such that the uniform estimates

$$
y_{i}(p)=x_{i}(p)-x_{i}\left(q_{1}\right) h(p)+0(\psi)
$$

$$
p \in M_{\varepsilon}, \quad 0<\varepsilon \leq \varepsilon_{1}, \quad i=1,2, \ldots, m
$$


are valid where $\psi(\varepsilon)=\varphi(\varepsilon)$ if $n=2$ and $\psi(\varepsilon)=\varepsilon$ if $n \geq 3$.

It is not our purpose to reproduce the entire proof here. To indicate some of the arguments, we shall deduce the first part of the theorem in the cases $n=2,3$ rather directly from some spectral estimation theory given by the writer in [6]. Let $A, A_{\varepsilon}$ be the linear integral operators whose kernels are the respective Green's functions $G(p, q), G_{\varepsilon}(p, q)$ associated with $\mathrm{M}, \mathrm{M}_{\varepsilon}$. The eigenvalues $\alpha, \alpha_{\varepsilon}$ a re known to be reciprocals of $\lambda, \mu$ respectively. Let $\mathrm{X}_{\alpha}$ be the eigenspace corresponding to the $\mathrm{m}$-fold degenerate eigenvalue $\alpha$, and let $\mathrm{X}_{\alpha \varepsilon}=\mathrm{P}_{\varepsilon} \mathrm{X}_{\alpha}$ where $\mathrm{P}_{\varepsilon}$ is the projection mapping from $L^{2}[\mathrm{M}]$ onto $\mathrm{L}^{2}\left[\mathrm{M}_{\varepsilon}\right]$. Clearly $\varepsilon_{0}$ can be chosen so that $\operatorname{dim} \mathrm{X}_{\alpha \varepsilon}=\operatorname{dim} \mathrm{X}_{\alpha}$ for $0<\varepsilon \leq \varepsilon_{\circ}$.

For $u \in X_{\alpha \varepsilon}$, the function $f=A_{\varepsilon} u-\alpha u$ is a solution of Lf $=0$ in $M_{\varepsilon}$ satisfying $f=-\alpha u$ on $Y_{\varepsilon}$. Let functions $g$ and $F$ be defined in $M_{\varepsilon}$ by the equations

$$
g(p)=\omega \varphi(\varepsilon) G\left(p, q_{1}\right), \quad F(p)=\left[2 \max _{\varepsilon}|f|\right] g(p)-f(p)
$$

where $\omega=2 \pi$ or $4 \pi$ according as $n=2$ or 3 . There is no loss of generality in supposing $\varepsilon$, has been selected so that $g(p) \geq 1 / 2$ for all $p \in \gamma_{\varepsilon}$ whenever $0<\varepsilon \leq \varepsilon_{0}$, because of the singula rity of $G\left(p, q_{1}\right)$ at $p=q_{1}$.

$$
\text { Since } L F=0 \text { in } M_{\varepsilon}, F=0 \text { on } B \text {, and } F \geq 0 \text { on } \gamma_{\varepsilon} \text {, }
$$
it follows from the maximum principle for elliptic differential equations [2, p. 102] that $F(p) \geq 0$ throughout $M_{\varepsilon}$. Then $f(p) \leq 2\left[\max _{y}|f|\right] g(p)$. A lower bound for $f(p)$ is established

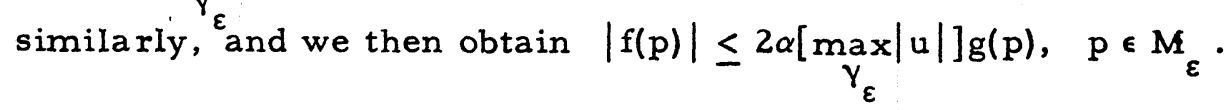

Then $\left\|A_{\varepsilon} u-\alpha u\right\|=\|f\| \leq c \varphi(\varepsilon)\|u\|$ for all $u \in X_{\alpha \varepsilon}$. Since 
$A_{\varepsilon}$ is a symmetric and completely continuous linear transformation on $L^{2}\left[M_{\varepsilon}\right]$, a known spectral estimation theorem $\left[6\right.$, p. 35] shows that at least $m$ eigenvalues $\alpha_{\varepsilon i}$ of $A_{\varepsilon}$ lie in the interval $[\alpha-c \varphi(\varepsilon), \alpha]$. It is well-known from the minimum-maximum principle for eigenvalues that $M_{\varepsilon} \subset M$ implies $\alpha_{n} \geq \alpha_{\varepsilon n}(n=1,2, \ldots)$. Then an easy induction proof establishes that the re are exactly $m$ eigenvalues in $[\alpha-c \varphi(\varepsilon), \alpha]$. This is equivalent to the first statement of theorem 2. The arguments used to prove the second part are similar to those used in [5] and will not be given here.

Theorem 2 will now be used to obtain the following special case of theorem 1 .

THEOREM 3. If $\lambda, \mu_{i}$ a re eigenvalues of (2.1), (4.1) and $x_{i}, y_{i}$ are corresponding normalized eigenfunctions, as described in theorem 2 , then the following a symptotic variational formulae a re valid:

$$
\mu_{i}-\lambda=\left[-\left|x_{i}\left(q_{1}\right)\right|^{2}+0(\psi)\right] \int_{\gamma_{\varepsilon}} \nabla h \cdot \underline{n} d S
$$

as $\varepsilon \rightarrow 0, i=1,2, \ldots, m$.

Proof. Since the L-measure has the property $\|h\|=0(\psi)$, it follows from (4.3) that $\left\|y_{i}-x_{i}\right\| \leq \delta(\varepsilon)\left\|x_{i}\right\|$, where $\delta(\varepsilon)=$ $\mathrm{c} \psi(\varepsilon), \quad 0<\varepsilon \leq \varepsilon_{1}$. Theorem 1 can then be applied provided $\varepsilon$ is on a positive interval $\left(0, \varepsilon_{0}\right]$ such that $0<\delta(\varepsilon) \leq \delta_{0}<1$. Since $\mu_{i}$ is real and $y_{i}$ vanishes on $\gamma_{\varepsilon}$, (3.1) reduces to

$$
\mu_{i}-\lambda=\int_{\gamma \varepsilon} x_{i} \nabla \bar{y}_{i} \cdot \underline{n} \mathrm{dS}[1+0(\psi)]
$$

We apply (2.2) to $h, y_{i}$ and $h, x_{i}$ in turn to obtain

$$
\mu_{i}\left(\dot{h}, y_{i}\right)=\int_{\gamma_{\varepsilon}} \nabla \bar{y}_{i} \cdot \underline{n} d S,
$$




$$
\lambda\left(h, x_{i}\right)=\int_{\gamma_{\varepsilon}}\left(h \nabla \bar{x}_{i}-\bar{x}_{i} \nabla h\right)^{\cdot} \underline{n} d S
$$

Use of (4.3), (4.6), and (4.7) yields

$$
\begin{aligned}
\int_{\gamma_{\varepsilon}} \nabla \bar{y}_{i} \cdot \underline{n} d S & =[\lambda+0(\varphi)]\left[\left(h, x_{i}\right)-(h, h) x_{i}\left(q_{1}\right)+(h, 1) 0(\psi)\right] \\
& =\lambda\left(h, x_{i}\right)+0\left(\psi^{2}\right) \\
(4.8) & =-\bar{x}_{i}\left(q_{1}\right)[1+0(\varepsilon)] \int_{\gamma_{\varepsilon}} \nabla h \cdot \underline{n} d S+0\left(\psi^{2}\right) .
\end{aligned}
$$

The result (4.4) then follows from (4.5) and (4.8).

As an example, consider the elliptic operator $L=I-\Delta$, where $I$ is the identity operator, on the unit 2-sphere. The metric is $\mathrm{ds}^{2}=\mathrm{d} \theta^{2}+\sin ^{2} \theta \mathrm{d} \varphi^{2}$, where $\theta, \varphi$ are the usual spherical polar angles. We select for $q_{1}$ the north pole $\theta=0$. Then $\gamma_{\varepsilon}$ is the closed curve $\theta=\varepsilon, 0 \leq \varphi \leq 2 \pi$ about $q_{1}$. The eigenvalues of the basic problem (2.1) are $\lambda_{m}=m^{2}-m+1$, $\mathrm{m}=1,2, \ldots$, which are $(2 \mathrm{~m}-1)$-degenerate. The corresponding normalized eigenfunctions are $x_{m i}=S_{m-1}^{i-1} /\left\|S_{m-1}^{i-1}\right\|$, where $\mathrm{S}_{\mathrm{m}-1}^{\mathrm{i}-1}$ are the spherical harmonics. It will be sufficient to consider the values $i=1,2, \ldots, m$. Thus $x_{m i}^{2}\left(q_{1}\right)=$ $(2 m-1) \delta_{i 1} / 4 \pi$ from the properties of Legendre functions, where $\delta_{i 1}$ is the Kronecker symbol, and (4.4) yields

$$
\mu_{m i}=m^{2}-m+1+\frac{1}{2}(2 m-1) \delta_{i 1}\left(\log \frac{1}{\varepsilon}\right)^{-1}+0\left[\left(\log \frac{1}{\varepsilon}\right)^{-2}\right] .
$$

If $i=2,3, \ldots, m$, the leading variational te $r m$ vanishes (i.e. $x_{m i}$ has a zero at $q_{1}$ ). The variables in the partial differential equation are separable in this example, and consideration of the associated Legendre ordinary differential equation by the methods of [3] or [4] leads to an asymptotic 
variation of order $\varepsilon^{2 i-2}$ if $i \geq 2$. It is left as an open question to decide if this is the general situation when $x$ has a zero of order i-1 at $q_{1}$.

5. Asymptotic variation under handle attachment. In this section, $\mathrm{N}_{\varepsilon}$ will be specialized to two open balls $\mathrm{N}_{\varepsilon 1}, \mathrm{~N}_{\varepsilon 2}$, with centres $q_{1} \in M, q_{2} \in M$ and boundaries $\gamma_{\varepsilon 1}, \gamma_{\varepsilon 2}$ respectively. For a fixed homeomorphism $h$ of $\gamma_{\varepsilon 1}$ into $\gamma_{\varepsilon}$, let points $p_{1} \in \gamma_{\varepsilon 1}$ and $p_{2} \in \gamma_{\varepsilon 2}$ be identified whenever $\mathrm{p}_{2}=\mathrm{h}\left(\mathrm{p}_{1}\right)$. The corresponding perturbed region $\mathrm{M}_{\varepsilon}^{*}$ consists of all points in $M_{\varepsilon}=M-\bar{N}_{\varepsilon}$ with the boundaries $Y_{\varepsilon 1}, Y_{\varepsilon} 2$ identified according to the rule $p_{2}=h\left(p_{1}\right)$. We assume that $h$ is an orientation-preserving homeomorphism. Thus $\mathrm{M}_{\varepsilon}^{*}$ is orientable along with $M$, and $\gamma_{\varepsilon 1}, \gamma_{\varepsilon 2}$ are oppositely oriented with respect to the common domain $M_{\varepsilon}$.

The perturbed domain $\mathrm{D}_{\varepsilon}^{*}$ is defined to be the set of all continuous complex-valued functions on the closure of $M_{\varepsilon}^{*}$ which are of class $\mathrm{C}^{2}\left[\mathrm{M}_{\varepsilon}^{*}\right]$ and zero on $B$. The perturbed eigenvalue problem for this domain is

$$
\text { Ly }=\mu y, \quad y \in D_{\varepsilon}^{*} \text {. }
$$

Instead of (4.2), the L-measure $h$ to be used in this section is the solution of the Dirichlet problem

$$
\begin{array}{ll}
(L h)(p)=0, & p \in M_{\varepsilon} ; \quad h(p)=0, \quad p \in B ; \\
h(p)=(-1)^{j}, & p \in Y_{\varepsilon j} \quad(j=1,2) .
\end{array}
$$

The following analogue of theorem 2 has been obtained by the write $r$ by a proof similar to that in [1].

THEOREM 4. The assertions of theorem 2 remain valid if (4.1) is replaced by (5.1) and (4.3) is replaced by 


$$
y_{i}(p)=x_{i}(p)-\frac{1}{2}\left[x_{i}\left(q_{2}\right)-x_{i}\left(q_{1}\right)\right] h(p)+0(\psi)
$$

The following is then obtained as the analogue of theorem 3 .

THEOREM 5. If $\lambda, \mu_{i}$ a re eigenvalues of (2.1), (5.1) and $x_{i}, y_{i}$ are corresponding normalized eigenfunctions, as described in theorem 4 , then

(5.4) $\mu_{i}-\lambda=\left[\frac{1}{2}\left|\mathrm{x}_{i}\left(\mathrm{q}_{2}\right)-\mathrm{x}_{\mathrm{i}}\left(\mathrm{q}_{1}\right)\right|^{2}+0(\psi)\right] \int_{\gamma_{\varepsilon 1}} \nabla \mathrm{h} \cdot \underline{\mathrm{n}} \mathrm{dS}$ as $\varepsilon \rightarrow 0, i=1,2, \ldots, m$.

Proof. With (5.1) instead of (4.1), (4.5) is replaced by $\mu_{i}-\lambda=\int_{\gamma_{\varepsilon}}\left(x_{i} \nabla \bar{y}_{i}-\bar{y}_{i} \nabla x_{i}\right) \cdot n d S[1+0(\psi)]$

Hence

$$
\begin{aligned}
\mu_{i}-\lambda & =\left[\mathrm{x}_{i}\left(\mathrm{q}_{1}\right)+0(\psi)\right] \int_{\gamma_{\varepsilon 1}} \nabla \overline{\mathrm{y}}_{\mathrm{i}} \cdot \underline{\mathrm{n}} \mathrm{dS} \\
& +\left[\mathrm{x}_{\mathrm{i}}\left(\mathrm{q}_{2}\right)+0(\psi)\right] \int_{\gamma_{\varepsilon}} \nabla \overline{\mathrm{y}}_{\mathrm{i}} \cdot \underline{\mathrm{n}} \mathrm{dS}+0\left(\varepsilon^{\mathrm{n}-1}\right) .
\end{aligned}
$$

The result (5.4) would follow if we knew that the order relation (5.3) could be differentiated. The actual proof of (5.4) is similar to that of theorem 3 and will be omitted.

In the example at the end of section 4 , if we take $q_{1}$ and $q_{2}$ to be the north and south poles respectively, then $x_{m 1}^{2}\left(q_{1}\right)=(2 m-1) / 4 \pi, \quad x_{m 1}\left(q_{2}\right)=(-1)^{m-1} x_{m 1}\left(q_{1}\right)$, and $(5.4)$ gives in particular

$\mu_{m 1}=m^{2}-m+1+\frac{1}{2}\left[1+(-1)^{m}\right](2 m-1)\left(\log \frac{1}{\varepsilon}\right)^{-1}+0\left[\left(\log \frac{1}{\varepsilon}\right)^{-2}\right]$, $\mathrm{m}=1,2, \ldots$ 


\section{REFERENCES}

1. H. F. Bohnenblust, C.R. DePrima, and C. A. Swanson, Elliptic operators with perturbed domains, in preparation.

2. G. F. D. Duff, Partial Differential Equations, (University of Toronto, 1956).

3. C. A. Swanson, Differential ope rators with perturbed domains, J. of Math. Mech. , 6 (1957) 823-846.

4. , On a non-analytic perturbation problem, Canad. Math. Bull. 4 (1961) 243-248.

5. - Asymptotic estimates for limit circle problems, Pacific J. Math., 11 (1961) 1549-1559.

6. , On spectral estimation, Bull. Amer. Math. Soc., 68 (1962) 33-35.

7. M. Schiffer and D.C. Spencer, Functionals of Finite Riemann Surfaces, (Princeton University, 1954).

The University of British Columbia 